\title{
Bladder filling variations during concurrent chemotherapy and pelvic radiotherapy in rectal cancer patients: early experience of bladder volume assessment using ultrasound scanner
}

\author{
Jee Suk Chang, MD, Hong In Yoon, MD, Hye Jung Cha, MD, Yoonsun Chung, MD, Yeona Cho, MD, \\ Ki Chang Keum, MD, Woong Sub Koom, MD \\ Department of Radiation Oncology, Yonsei University College of Medicine, Seoul, Korea
}

\begin{abstract}
Purpose: To describe the early experience of analyzing variations and time trends in bladder volume of the rectal cancer patients who received bladder ultrasound scan.

Materials and Methods: We identified 20 consecutive rectal cancer patients who received whole pelvic radiotherapy (RT) and bladder ultrasound scan between February and April 2012. Before simulation and during the entire course of treatment, patients were scanned with portable automated ultrasonic bladder scanner, 5 times consecutively, and the median value was reported. Then a radiation oncologist contoured the bladder inner wall shown on simulation computed tomography (CT) and calculated its volume. Results: Before simulation, the median bladder volume measured using simulation CT and bladder ultrasound scan was 427 $\mathrm{mL}$ (range, 74 to $1,172 \mathrm{~mL}$ ) and $417 \mathrm{~mL}$ (range, 147 to $1,245 \mathrm{~mL}$ ), respectively. There was strong linear correlation $(\mathrm{R}=0.93, \mathrm{p}<$ 0.001 ) between the two results. During the course of treatment, there were wide variations in the bladder volume and every time, measurements were below the baseline with statistical significance (12/16). At 6 weeks after RT, the median volume was reduced by $59.3 \%$ to $175 \mathrm{~mL}$. Compared to the baseline, bladder volume was reduced by $38 \%$ or $161 \mathrm{~mL}$ on average every week for 6 weeks.

Conclusion: To our knowledge, this study is the first to prove that there are bladder volume variations and a reduction in bladder volume in rectal cancer patients. Moreover, our results will serve as the basis for implementation of bladder training to patients receiving RT with full bladder.
\end{abstract}

Keywords: Rectal neoplasms, Urinary bladder, Ultrasonography, Observer variation, Radiotherapy

\section{Introduction}

Preoperative chemoradiotherapy (CRT) has been established as a current standard treatment on locally advanced rectal cancer since several randomized controlled trials have confirmed that it improves local control and increases sphincter preservation rate [1-4]. Even after an upfront surgery of localized rectal cancer, for tumors with adverse pathological features (T3-4 or $\mathrm{N}+$ tumors), adjuvant CRT is known to improve oncologic outcome $[5,6]$. For rectal cancer patients, CRT on pelvic area is generally tolerable on most of the patients, but $8-40 \%$ of patients are reported to have acute toxicity (grade $\geq 3$ ) and

Received 22 January 2013, Revised 12 February 2013, Accepted 26 February 2013.

Correspondence: Woong Sub Koom, MD, Department of Radiation Oncology, Yonsei Cancer Center, Yonsei University College of Medicine, 50 Yonsei-ro, Seodaemun-gu, Seoul 120-752, Korea. Tel: +82-2-2228-8116, Fax: +82-2-312-9033, E-mail: mdgold@yuhs.ac

(c) This is an Open Access article distributed under the terms of the Creative Commons Attribution Non-Commercial License (http://creativecommons.org/ licenses/by-nc/3.0/) which permits unrestricted non-commercial use, distribution, and reproduction in any medium, provided the original work is properly cited.

www.e-roj.org 
$24 \%$ of patients with late toxicity $[1,7,8]$.

It is known that the risk of gastrointestinal (GI) complications is closely related to irradiated small bowel volume and dose within the radiation (RT) field [9]. There have been many studies to minimize irradiated small bowel volume and dose. Drzymara et al. [10] and Nijkamp et al. [11] reported that prone position was more effective than supine position in sparing small bowel. In the case of patients who are treated in prone position, using belly board or small bowel displacement device can move small bowel out of the RT field and, as a result, can lower the small bowel dose $[12,13]$. Therefore, in order to reduce $\mathrm{Gl}$ complication risk in rectal cancer patients, our institution has been instructing all patients to maintain full bladder and treating them with belly board in prone position since 2009.

However, to date, no study on whether rectal cancer patients have been maintaining the same bladder volume during the

Table 1. Clinical profiles of 20 patients

\begin{tabular}{lc}
\hline \multicolumn{1}{c}{ Characteristic } & No. (\%) \\
\hline Age (yr), median (range) & $50(40-75)$ \\
Gender & $10(50)$ \\
Female & $10(50)$ \\
Male & $1(5)$ \\
ECOG performance status & $19(95)$ \\
0 & \\
1 & $8(40)$ \\
Tumor location & $11(55)$ \\
Lower rectum & $1(5)$ \\
Mid rectum & \\
Upper rectum & $1(5)$ \\
Clinical T stage & $13(65)$ \\
T2 & $6(30)$ \\
T3 & \\
T4 & $4(20)$ \\
Clinical N stage & $16(80)$ \\
N0 & \\
N1 & $20(100)$ \\
Clinical M stage & \\
M0 & $1(5)$ \\
Clinical stage & $2(10)$ \\
I & $1(5)$ \\
IIA & $12(60)$ \\
IIC & $4(20)$ \\
IIIB & $50.4(45-59.4)$ \\
IIIC & $28(25-33)$ \\
RT dose (Gy), median (range) & \\
RT fraction, median (range) &
\end{tabular}

ECOG, the Eastern Cooperative Oncology Group; RT, radiotherapy.
5 to 6 weeks of RT as they were during simulation has been published. Studies of bladder volume of cervix cancer and prostate cancer patients not only show wide inter-fraction variations in bladder volume during each RT but even a decrease in bladder volume during the course of treatment $[14,15]$. Therefore, in order to check bladder volume on a day by day basis just prior to RT, our institution introduced ultrasound bladder ultrasound scan in February 2011. The purpose of this study is to describe the early experience of analyzing variations and time trends in bladder volume of the 20 consecutive rectal cancer patients who received bladder ultrasound scan after the introduction of the device.

\section{Materials and Methods}

\section{Study population}

We identified 20 consecutive rectal cancer patients who received whole pelvic $R T$ and bladder ultrasound scan between February 14 and April 15, 2012. The screening was performed after obtaining informed consent from all patients. Patients with the Eastern Cooperative Oncology Group (ECOG) performance status of 2 or higher, those who had functional disorder in their bladder, patients who underwent cystectomy, or those who showed strong aversion to retaining urine were excluded from bladder ultrasound scan. The clinical profiles of the 20 patients are shown in Table 1.

\section{Treatment}

Fourteen patients (70\%) received RT for preoperative aim and 6 patients (30\%) as postoperative aim. All patients received 5 -fluorouracil based concurrent chemotherapy plus RT. The former group comprised of patients with stage III mid-lower rectal cancer. One of the 6 patients in the latter group had stage I cancer and had received local excision before the CRT, while the other 5 patients had stage IIIB cancer and had undergone laparoscopy or robot assisted low anterior resection (LAR). Preoperative CRT patients received whole-pelvic RT of $45 \mathrm{~Gy}$ followed by tumor bed boost of $5.4 \mathrm{~Gy}$. On the other hand, Postoperative CRT patients only received whole-pelvic RT of $45 \mathrm{~Gy}$, and additional boost of RT was given only according to their resection margin status.

\section{Bladder volume evaluation}

Patients were asked to drink unspecified volume of water because we thought there were wide variations of abilities in drinking water and retaining their urine. Instead, patients 
were instructed voluntarily at their first visit to regulate their volume of drinking water and time of retaining the urine so that the goal of the instructions was maintaining the similar desire to micturition before treatment. However, the patients were not forced to follow this protocol if they were not comfortable. During the simulation, patients were in prone position using bladder compression device and belly board

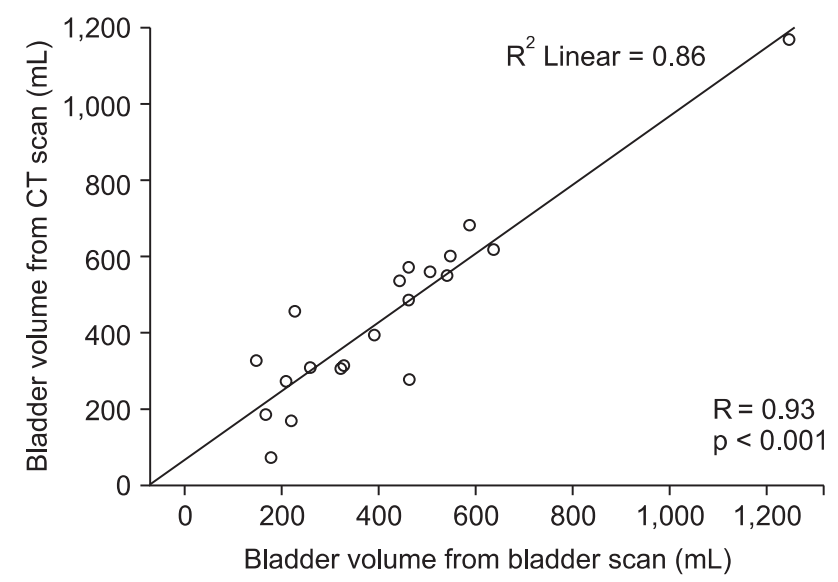

Fig. 1. A Pearson correlation scatter plot for 20 patients between the two different bladder volume detection methods. CT, computed tomography. designed by our institution. Details are included in previously reported paper [16]. Two trained doctors controlled the portable automated ultrasonic bladder scanner (Biocon-700; Mcube Technology, Seoul, Korea). Patients lied supine in a comfortable position. Then, scanner's probe was placed on the midline of abdomen, two fingers over the symphysis pubic bone and angled towards the bladder. A three-dimensional real-time image before scanning was displayed to operators in order to locate easily the bladder position for more accurate measurement. Before simulation computed tomography (CT), patients were scanned 5 times consecutively, and the median value was reported. Then a radiation oncologist contoured the bladder inner wall shown on simulation CT and calculated its volume. During the entire course of treatment, patients bladder was scanned 3 days weekly (Monday, Wednesday, and Friday) just prior to the RT, and just as mentioned above, patients were scanned 5 times before reporting the median value. No additional planned instructions were provided to patients after full education at first visit. All measured volumes by bladder ultrasound scan were blinded to patients.

\section{Data and statistical analysis}

In order to assess the accuracy of bladder ultrasound scan,

Table 2. Time trends of bladder volume from CT scans and bladder scans

\begin{tabular}{|c|c|c|c|c|c|}
\hline & No. of patient & Median (mL) & IQR (mL) & Range $(\mathrm{mL})$ & $p$-value \\
\hline$V_{C T}$ & 20 & 427 & 291 & $74-1,172$ & - \\
\hline$V_{\text {B-SCAN }}$ & 20 & 417 & 222 & $147-1,245$ & - \\
\hline$V_{1-1 \text { week }}$ & 19 & 278 & 193 & $77-660$ & 0.053 \\
\hline$V_{1-2 \text { week }}$ & 18 & 269 & 173 & $78-735$ & 0.008 \\
\hline$V_{1-3 \text { week }}$ & 15 & 211 & 124 & $34-574$ & 0.005 \\
\hline$V_{2-1 \text { week }}$ & 17 & 219 & 132 & $80-572$ & 0.019 \\
\hline$V_{2-2 \text { week }}$ & 19 & 188 & 141 & $58-1,028$ & 0.053 \\
\hline$V_{2-3 \text { week }}$ & 18 & 258 & 175 & $110-1,002$ & 0.061 \\
\hline$V_{3-1 \text { week }}$ & 20 & 299 & 169 & $35-869$ & 0.055 \\
\hline$V_{3-2 \text { week }}$ & 19 & 255 & 153 & $21-513$ & 0.049 \\
\hline$V_{3-3 \text { week }}$ & 19 & 261 & 157 & $42-677$ & 0.036 \\
\hline$V_{4-1 \text { week }}$ & 19 & 253 & 177 & $37-797$ & 0.046 \\
\hline$V_{4-2 \text { week }}$ & 19 & 263 & 138 & $35-579$ & 0.049 \\
\hline$V_{4-3 \text { week }}$ & 19 & 315 & 176 & 73-852 & 0.126 \\
\hline$V_{5-1 \text { week }}$ & 19 & 308 & 190 & $122-643$ & 0.033 \\
\hline$V_{5-2 \text { week }}$ & 17 & 228 & 165 & 19-980 & 0.006 \\
\hline$V_{5-3 \text { week }}$ & 16 & 192 & 145 & $66-652$ & 0.004 \\
\hline$V_{6-1 \text { week }}$ & 16 & 188 & 109 & $50-917$ & 0.001 \\
\hline$V_{6-2 \text { week }}$ & 10 & 150 & 124 & $109-210$ & 0.013 \\
\hline$V_{6-3 \text { week }}$ & 5 & 174 & 121 & $62-257$ & 0.138 \\
\hline
\end{tabular}

IOR, interquartile range; $C T$, computed tomography; $V_{C T}$, bladder volume measured from simulation $C T$ scans; $V_{B-S c A N}$, bladder volume scanned by bladder scan prior to simulation CT scans; $V_{x-x \text { week, }}$ bladder volume scanned by bladder scan at post-RT $x-x$ week. 
the bladder volumes contoured during the simulation CT and the baseline bladder volumes measured through the bladder ultrasound scan before the simulation CT were compared using Pearson correlation test. The bladder volumes measured during the RT and the baseline bladder volumes were compared using Wilcoxon signed rank test. Absolute and relative bladder volume changes (from the baseline) of all patients were also calculated each time. Then, using the median values of the measurements and interquartile range (IOR), a graph was drawn. It was defined statistically significant when $p<0.05$ and SPSS ver. 20.0.0 (IBM, Armonk, NY, USA) was used.

\section{Results}

\section{Measurement of bladder volume}

Twenty patients were scheduled to receive bladder ultrasound scans at a median number of 18 times (range, 15 to 19 times; 3 days a week according to the RT schedule) in total. Ninety percent of the scans were carried out as planned, and patients had scans at a median number of 16 times (range, 12 to 19 times) in total. The median bladder volume measured using simulation CT was $427 \mathrm{~mL}(\mathrm{IQR}, 291 \mathrm{~mL}$; range, 74 to 1,172 $\mathrm{mL}$ ). The median volume measured using bladder ultrasound scan before the simulation CT on the same day was $417 \mathrm{~mL}$ (IOR, $222 \mathrm{~mL}$; range, 147 to 1,245 mL). There was strong linear correlation $\left(R=0.93, p<0.001 ; R^{2}=0.86, p<0.001\right.$ ) between the two results (Fig. 1). The bladder volume measurements and the number of patients are recorded in Table 2.

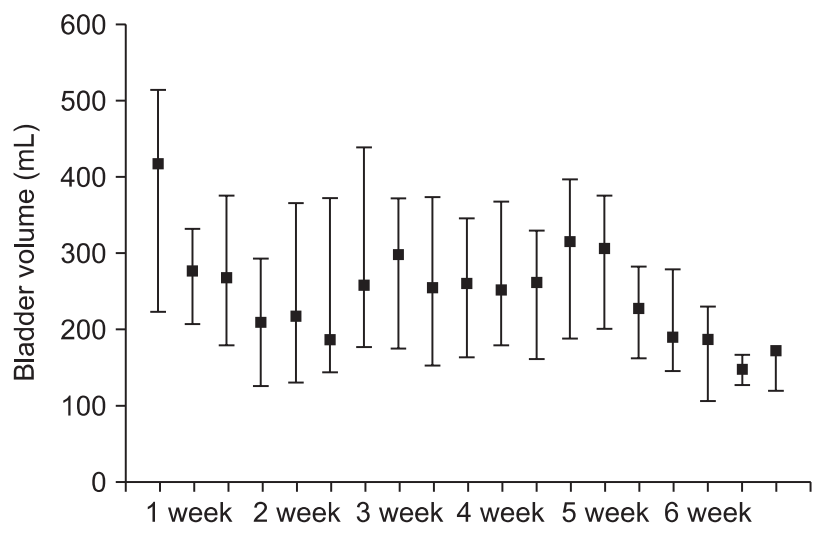

Fig. 2. Bladder volume measured by bladder scan over the course of treatment. Error bars represent the median \pm interquartile range.

\section{Time trends of bladder volume}

The time trends of bladder volume were depicted in Fig. 2. Measured volumes during RT were always below the baseline volume, and most were of statistical significance (12/16) or borderline significance $(3 / 16)$. On the third day of the third week (3-3 weeks) after the RT, the median volume decreased by $38.9 \%$ to $261 \mathrm{~mL}(I 0 R, 157 \mathrm{~mL})$, and at by the third day of the sixth week (6-3 weeks), it was reduced by $59.3 \%$ to 175 $\mathrm{mL}(I \mathrm{R}, 121 \mathrm{~mL})$. The relative and absolute bladder volume changes from each patient's baseline values are displayed in Figs. 3 and 4. Compared to the baseline, bladder volume was reduced by $38 \%$ (standard deviation [SD], 14\%) or $161 \mathrm{~mL}$ $(S D, 53 \mathrm{~mL})$ on average every week for 6 weeks. To see the intrapatient variation, we calculated overall mean and SD of

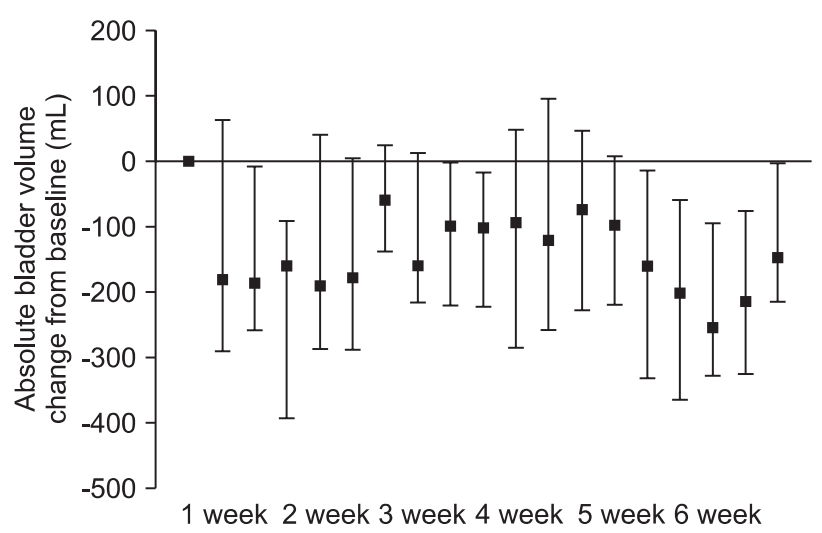

Fig. 3. Absolute bladder volume change from baseline over the course of treatment. Error bars represent the median \pm interquartile range.

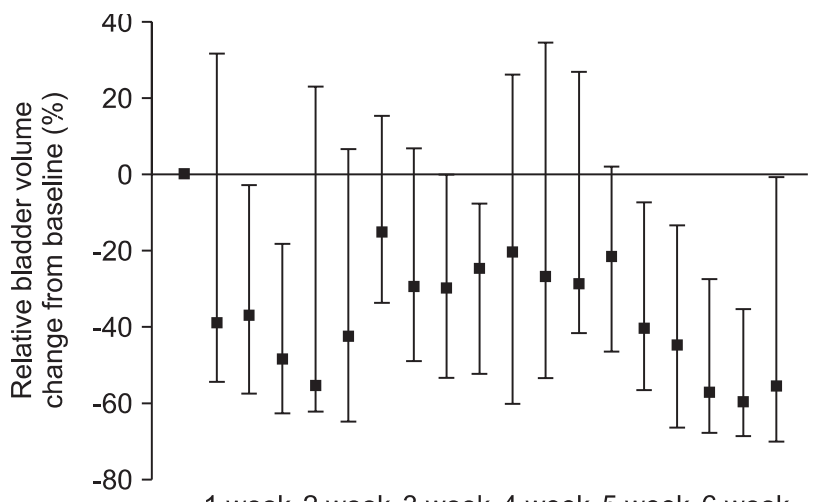

1 week 2 week 3 week 4 week 5 week 6 week

Fig. 4. Relative bladder volume change from baseline over the course of treatment. Error bars represent the median \pm interquartile range. 
each patient's standard deviation (mean \pm SD, $155 \pm 100 \mathrm{~mL}$ ).

\section{Discussion and Conclusion}

This study demonstrated that during pelvic RT of rectal cancer patients with full bladder, there are wide variations in bladder volume as well as significant reduction in bladder volume from the baseline. As far as we are aware, this study is the first to analyze variations in bladder volume and time trend during RT in patients with rectal cancer.

There have been many studies on the accuracy of bladder ultrasound scan $[15,17,18]$. Ahmad et al. [15] used bladder phantom to prove that bladder volume measured with CT or bladder ultrasound scan agrees with real bladder volume. It also reported that the difference between measurements of 4 healthy volunteers' bladder volume before and after urination equaled to actual urination volume. Although this study also showed strong positive correlation $(R=0.89)$, there is a slight difference from other studies. This may be due to the time gap between CT scan and bladder ultrasound scan, which range from as little as a few minutes to as much as an hour. In order to minimize such error when evaluating the accuracy of bladder ultrasound scan, two bladder ultrasound scans (before and after CT scan) may be necessary.

With regards to prostate cancer, there have been many studies reported on bladder volume $[11,14,18,19]$. Nakamura et al. [14] reported that measurements of bladder volume during RT using megavoltage $\mathrm{CT}$ over the course of treatment showed $38 \%$ of mean volume reduction from the baseline at the end of treatment $(p<0.001)$. Other studies that used daily or weekly bladder ultrasound scans reported $16-50 \%$ of volume reduction from the baseline $[6,11,18]$. In the case of cervix cancer, Ahmad et al. [15] and other studies that used bladder ultrasound scan reported an average of $71 \%$ of volume reduction. In our study, there was 59\% reduction, which is roughly consistent with the results from prostate cancer studies. We also observed the bladder volume was already decreased at the first week of treatment (median, $417 \mathrm{~mL}$ ) when compared to those at the time of simulation as baseline (211 to $278 \mathrm{~mL}$ ). The most plausible explanation is that the full bladder instruction at the first visit alone was not sufficient to maintain the patient's compliance until and though treatment (the interval between first visit and treatment start was generally one week).

That bladder volume is not reproducible and rather reduces every time means that RT dose on organ at risk could be higher than planned. Therefore, efforts to minimize the reduction of bladder volume are needed. Stam et al. [17] and O'Doherty et al. [18] showed that providing labor-intensive education to patients could help maintain bladder volume consistency. O'Doherty et al. [18] instructed 41 patients to drink $350 \mathrm{~mL}$ of water and retain their urine for an hour and a half, and then asked them about their bladder fullness. Only 25 of them were given patient information sheet, and the bladder volumes of the two groups were compared. Patient information sheet was developed by a multidisciplinary team, and it contained the information regarding the importance of full bladder, how to achieve a comfortably full bladder successfully, and how to manage if the patient could not hold the full bladder. Patients without the information sheet showed a significant reduction in bladder volume (mean, $362 \mathrm{~mL} \rightarrow 251 \mathrm{~mL}$ ), but the other group maintained consistency (mean, $286 \mathrm{~mL} \rightarrow 312$ $\mathrm{mL}$ ). Stam et al. [17] provided bio-feedback to only 18 of 34 patients and not for the remaining 16 patients, then compared to two groups. To elaborate, if daily measurements of bladder volume were below $80 \%$ or above $120 \%$ of the baseline bladder volume, patients were given negative feedback, and if the measurements were between $80 \%$ and $120 \%$, they were given positive feedback (drink the same amount of water the next day). The group with bio-feedback showed 7\% improvement. Our institution is also planning to develop ways to educate and perform prospective evaluations on patients in order to raise rectal cancer patients' compliance to full bladder instruction during the course of treatment.

There are several limitations to this study; therefore, interpreting the results requires some caution. First of all, although the study was a retrospective non-interventive observation study, there could have been unexpected biofeedback in the process of bladder ultrasound scan, which could have affected the result. Secondly, this study included not only patients who underwent preoperative RT but also 6 patients who received postoperative RT. Patients who had underwent an operation could have had bowel adhesion and showed different complications after receiving $R T$, which could have affected the result as well. Even so, this study is the first to prove that there are bladder volume variations and a reduction in bladder volume in not only prostate and cervix cancer but also rectal cancer patients. Moreover, our results will serve as the basis for implementation of bladder training to patients receiving RT with full bladder.

Our institution plans to continue studying in order to develop the optimal bladder ultrasound scan schedule and patient 
education method, and ultimately find effective intervention methods by identifying patients with wide bladder volume variations or those at high risk of bladder volume reduction. Moreover, compared to three-dimensional conformal RT, further study of bladder filling will be necessary for the pelvic intensity modulated RT that requires longer treatment time and different dose distribution to the organ at risks.

In conclusion, there were wide variations in bladder volume during treatment between rectal cancer patients who received concurrent chemotherapy and those who were treated with pelvic RT. Moreover, there was significant reduction from the baseline volume. This study was able to measure the bladder volume in a non-invasive manner using ultrasound-bladder scanner.

\section{Conflict of Interest}

No potential conflict of interest relevant to this article was reported.

\section{Acknowledgments}

Thanks to Wonmi Nam for her valuable help. This work was supported by Seoul Metropolitan Government, Seoul Business Agency (2012).

\section{References}

1. Sauer $R$, Becker $H_{1}$ Hohenberger W, et al. Preoperative versus postoperative chemoradiotherapy for rectal cancer. N Engl J Med 2004;351:1731-40.

2. Peeters KC, Marijnen CA, Nagtegaal ID, et al. The TME trial after a median follow-up of 6 years: increased local control but no survival benefit in irradiated patients with resectable rectal carcinoma. Ann Surg 2007;246:693-701.

3. Sebag-Montefiore D, Stephens RJ, Steele R, et al. Preoperative radiotherapy versus selective postoperative chemoradiotherapy in patients with rectal cancer (MRC CRO7 and NCIC-CTG C016): a multicentre, randomised trial. Lancet 2009;373:811-20.

4. Sauer $R$, Liersch T, Merkel $S$, et al. Preoperative versus postoperative chemoradiotherapy for locally advanced rectal cancer: results of the German CAO/ARO/AIO-94 randomized phase III trial after a median follow-up of 11 years. J Clin Oncol 2012;30:1926-33.

5. Colorectal Cancer Collaborative Group. Adjuvant radiotherapy for rectal cancer: a systematic overview of 8,507 patients from
22 randomised trials. Lancet 2001;358:1291-304.

6. Douglass HO Jr, Moertel CG, Mayer RJ, et al. Survival after postoperative combination treatment of rectal cancer. N Engl J Med 1986;315:1294-5.

7. Roh MS, Colangelo LH, O'Connell MJ, et al. Preoperative multimodality therapy improves disease-free survival in patients with carcinoma of the rectum: NSABP R-03. J Clin Oncol 2009;27:5124-30.

8. Gerard JP, Conroy T, Bonnetain F, et al. Preoperative radiotherapy with or without concurrent fluorouracil and leucovorin in T3-4 rectal cancers: results of FFCD 9203. J Clin Oncol 2006; 24:4620-5.

9. Kavanagh BD, Pan CC, Dawson LA, et al. Radiation dosevolume effects in the stomach and small bowel. Int J Radiat Oncol Biol Phys 2010;76(3 Suppl):S101-7.

10. Drzymala M, Hawkins MA, Henrys AJ, et al. The effect of treatment position, prone or supine, on dose-volume histograms for pelvic radiotherapy in patients with rectal cancer. Br J Radiol 2009;82:321-7.

11. Nijkamp J, Doodeman B, Marijnen C, Vincent A, van VlietVroegindeweij C. Bowel exposure in rectal cancer IMRT using prone, supine, or a belly board. Radiother Oncol 2012;102:229.

12. Kim TH, Chie EK, Kim DY, et al. Comparison of the belly board device method and the distended bladder method for reducing irradiated small bowel volumes in preoperative radiotherapy of rectal cancer patients. Int J Radiat Oncol Biol Phys 2005;62:769-75.

13. Park W, Huh SJ, Lee JE, et al. Variation of small bowel sparing with small bowel displacement system according to the physiological status of the bladder during radiotherapy for cervical cancer. Gynecol Oncol 2005;99:645-51.

14. Nakamura N, Shikama N, Takahashi O, et al. Variability in bladder volumes of full bladders in definitive radiotherapy for cases of localized prostate cancer. Strahlenther Onkol 2010;186:637-42.

15. Ahmad R, Hoogeman MS, Quint S, Mens JW, de Pree I, Heijmen BJ. Inter-fraction bladder filling variations and time trends for cervical cancer patients assessed with a portable 3dimensional ultrasound bladder scanner. Radiother Oncol 2008;89:172-9.

16. Yoon $\mathrm{HI}$, Chung $\mathrm{Y}$, Kim JH, et al. The efficacy of the change in belly board aperture location by the addition of bladder compression device for radiotherapy of rectal cancer. J Korean Soc Ther Radiol Oncol 2010;28:231-7.

17. Stam MR, van Lin EN, van der Vight LP, Kaanders JH, Visser AG. Bladder filling variation during radiation treatment of prostate cancer: can the use of a bladder ultrasound scanner and biofeedback optimize bladder filling? Int J Radiat Oncol 
Biol Phys 2006;65:371-7.

18. O'Doherty UM, McNair HA, Norman AR, et al. Variability of bladder filling in patients receiving radical radiotherapy to the prostate. Radiother Oncol 2006;79:335-40.
19. Hynds S, McGarry CK, Mitchell DM, et al. Assessing the daily consistency of bladder filling using an ultrasonic Bladderscan device in men receiving radical conformal radiotherapy for prostate cancer. Br J Radiol 2011;84:813-8. 